\title{
MIXED DISCRETE-EVENTS AND AGENT-BASED MODELING OF A COMPLEX AUTOMATIC WAREHOUSE FOR COPPER TUBES BUNDLES
}

\author{
Braglia Marcello $^{(a)}$, Frosolini Marco*(b), Gabbrielli Roberto ${ }^{(\mathrm{c})}$, Marrazzini Leonardo ${ }^{(\mathrm{d})}$, Padellini Luca $^{(\mathrm{e})}$ \\ (a), (b), (c), (d) Dipartimento di Ingegneria Civile e Industriale - Università di Pisa, Largo Lucio Lazzarino 2, 56126 Pisa \\ (Italy) \\ (e) Dipartimento di Ingegneria dell'Informazione - Università di Pisa, Via Girolamo Caruso 16, 56122 Pisa (Italy) \\ (a) m.braglia@ing.unipi.it, ${ }^{(b)}$ marco.frosolini@,ing.unipi.it, ${ }^{(\mathrm{c})}$ r.gabbrielli@ing.unipi.it, \\ (d) leonardo.marrazzini@ing.unipi.it \\ (e) $\underline{\text { luca.padellini@phd.unipi.it }}$ \\ *corresponding author
}

\begin{abstract}
The use of simulation modelling in science and engineering is today well established. Three methods exist that allow to build complex simulation models in many different contexts: Discrete Event Simulation, System Dynamics, and Agent Based Simulation. Despite the interesting results obtained, what has really limited the parallel and combined application of the three currently used paradigms was the lack of integrated simulation tools. This paper presents the outcomes of a complex plant simulation that has been carried out by means of the parallel and combined application of Discrete Event Simulation and Agent Based Simulation, while using a well-structured modular approach. This presents the advantage of simplifying the building, the debugging and the validation of the whole simulation model, while, at the same time, it offers the unquestionable benefit of allowing a widespread reusability of the simulation code.
\end{abstract}

Keywords: Industry 4.0; Discrete event simulation; Agents; Object-oriented programming.

\section{INTRODUCTION}

In the Industry 4.0 era, simulation modelling is a growing and attractive solution to reduce costs, shorten development cycles, increase the quality of products and processes, and greatly facilitate knowledge management. Nowadays, the use of simulation modelling in science and engineering is well established. A great body of scientific and professional literature on various aspects of Simulation modelling, especially in an object-oriented environment, is available, from original works such as Zeigler (1987) and Zeigler (1990) to newer publications, for example Piccinini et al. (2018). Among the simulation techniques, Discrete Event Simulation, System Dynamics, and Agent Based Simulation, are probably the most used and discussed. The recent framework of Sumari, Ibrahim, Zakaria, and Hamid (2013) compares all these three methods in terms of features, advantages, disadvantages and tools being used in each simulation method.
Despite the interesting results obtained, what has really limited the parallel and combined application of the three currently used paradigms was the lack of integrated simulation tools. In response, this paper presents the outcomes of a significantly complex plant simulation that has been carried out by means of the parallel and combined application of Discrete Event Simulation and Agent Based Simulation, while using a well-structured modular approach. Whilst the method is not new at all, as many authors have stated (Zeigler 1987, Zeigler 1990), its importance over time since the last decade of the last century, the availability of improved simulation tools makes it easily usable today.

The remaining part of this paper is organized as follows. Section 2 presents the literature review. In Section 3, we deeply explain the purpose of the study and its related approach. In order to show the operating principles and potential results of this novel approach, a real industrial implementation is presented in Section 4. Section 5 introduces and explains the verification and the validation of the model. In the last section, we summarise the key findings of the study and suggest future research directions.

\section{LITERATURE REVIEW}

The efficiency and effectiveness of logistics activities and of distribution networks in particular is largely determined by the operation of the nodes of the network, i.e. the warehouses. Warehouses are important links in the supply chain; here, products are temporarily stored and retrieved subsequently from storage locations to fulfil customer' orders (Rouwenhorst et al. 2000).

Assessing the performance of a warehouse in terms of cost, throughput, space utilization, and service provides feedback about how a specific design or operational policy performs compared with the requirements, and how it can be improved (Calzavara et al. 2017, Guo, Yu, and De Koster 2015). Furthermore, a good performance evaluation model can help the designer to quickly evaluate many design alternatives and narrow down the design space during the early design stage. Performance evaluation methods include benchmarking, analytical 
models, and simulation models (Gu, Goetschalckx, and McGinnis 2010). However, this should not obscure the fact that simulation is, nowadays, the most widely used technique for warehouse performance evaluation in the academic literature as well as in practice.

Specifically, simulation models have recently appeared as useful tools for analyzing specific issues related to warehouses in terms of, among the others, inventory management, logistics and layout design (Krenczyk, Davidrajuh, and Skolud 2019; Smith and Srinivas 2019; Vieira et al. 2018). The main reason for adopting simulation is that, in many practical cases, the definition of exact analytic models for managing a warehouse could be a complex task, especially when numerous factors, such as inventory policies, number of items, physical layout should be considered.

On the basis of the premise above, our study tries to contribute to the literature on "warehouse simulation modelling" in the following ways:

- To begin with, this paper presents the outcomes of a significantly complex plant simulation that has been carried out by means of the parallel and combined application of Discrete Event Simulation, System Dynamics and Agent Based Simulation, while using a well-structured modular approach. Specifically, a very complex model dealing with the automatic warehouse management of a plant for copper tubes production, has been effectively decomposed into a hierarchical, modular structure that is easy to develop, test, validate, modify and maintain.

- The single components that constitute the model can be effortlessly adapted to different situations and, thanks to a fully parametric approach, they can be effectively used to simplify the modelling phase and to optimize the model itself.

\section{THE PURPOSE OF THE WORK AND APPROACH}

As stated above, in the paper we present a combined approach which presents the advantage of simplifying the building and the debugging of the whole simulation model, while, at the same time, it offers the unquestionable benefit of allowing a widespread reusability of the simulation code. Indeed, during the initial assessment of the real-world application under analysis, that will be briefly described in the following, two major key points emerged clearly:

1. The complete process presents a great complexity, due to the large number of physical elements, rules and exceptions, that characterize the different material flows.

2. The actual model presents many repeated subprocesses (with limited or no modifications) and similar equipment and facilities.
The first point testifies the necessity of opportunely divide the simulation into smaller pieces of code, that can be put together when needed to build the complete picture. Small pieces mean, after all, less errors and easy maintenance of the simulation model, in particular with respect to the testing phase, as well as future enhancements and modifications. The presence of many commonalities makes this method even more convenient, in that many small pieces of code can be easily reused and adapted to different parts of the model itself. Further, the adoption of the agent modelling approach permits to transform each single sub-model into a standalone, autonomous and fully recyclable object. Indeed, by means of a consistent amount of opportunely chosen parameters, the single modules can be copied, pasted and set up in place with few modifications, making the model construction fast and straightforward. We could almost venture a similitude with the well-known "plug \& play" systems.

Therefore, while the discrete events modelling still continues to constitute the backbone of the whole simulation model, small object-oriented autonomous agents are used both to characterize the products flowing within the plant, the orders and the equipment (cranes, shuttles, machines and stores, for example) as well as the flows themselves.

The inner structure of each single agent is in turn characterized by a varied mix of simulation techniques. Again, discrete events have been widely used to represents logic flows, when applicable. On the contrary, some other logic behaviors have been extensively modelled by means of state charts (often, making the most of the communication skills of the single agents) and pure object-oriented java programming techniques. The final outcome is a complete set of reusable components that can be linked together within the main application body.

It can be said, briefly, that hundreds different objects have been correctly and effectively reproduced by means of no more than a dozen of agents. Owing to this, the main body of the simulation model acts as a true control system, that addresses the different products to the correct destinations, whereas the management of the single sub-processes is completely delegated to the above mentioned quasi-intelligent agents.

Specificities, that often occur within the real-world application, can be easily introduced within the simulation by making the most of the Object-oriented programming (OOP) linked concepts of inheritance and polymorphism (Armstrong 2006, Kindler and Krivy 2011). Indeed, an entire sub-model can be derived to build a new entity, that inherits the complete functionalities of the ancestor and that can be subsequently modified and adapted to specific requirements.

As already mentioned, the validation stage, usually difficult and cumbersome, and certainly highly timeconsuming, can be reduced consistently both in terms of complexity and duration, in that each single agent needs to be tested only once. Besides, small pieces of code are 
easier to be effectively validated with respect to a large, monolithic application

\section{THE INDUSTRIAL CASE STUDY}

The studied model mimics the behavior of a complex, high-intensity automatic warehouse where thousands copper tubes (having different lengths, weights and characteristics) are stored, handled and moved each day. The warehouse represents the main buffer between the production site, constituted by a foundry and a drawing plant, and the outgoing interface, where heavy load trucks are prepared continuously, in order to serve customers all over the world (Figure 1).

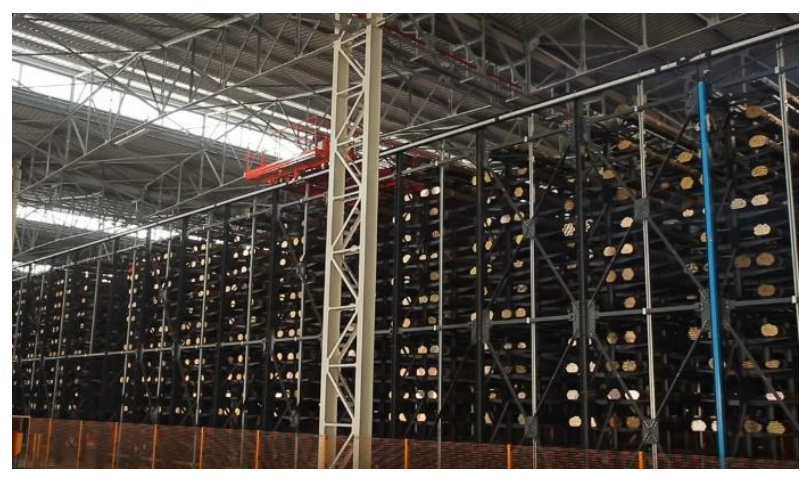

Figure 1: The automatic bundles warehouse

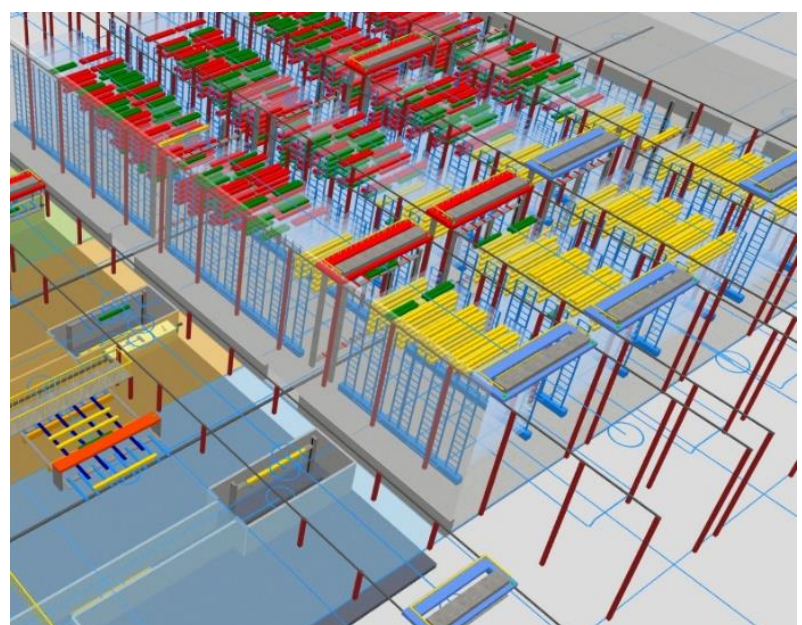

Figure 2: Simulation model of the automatic bundles warehouse

Products exiting the drawing plant are moved towards the warehouse, that is several meters far from the production site, by means of a few underground shuttles (whose end stations are partially visible in the simulated 3D model of Figure 2). Loading and unloading of the shuttles is performed by means of dedicated lifters and, in order to reduce the transferral time in presence of extraordinarily long paths, intermediate stations and duplicate shuttles may be present. The incoming tube bundles are then loaded by two different middle-height cranes, depending on the incoming lane and the destination location, that serve as distributors for the following stations (their number may vary depending on the site dimensions). These, in turn, are used to control the bundles, removing defective elements prior to store and expedite them, and to send them to the warehouse shelves by means of a different set of ground shuttles. The high intensity warehouse is constituted by a variable number of parallel shelves (about 10 meters high, with double depth and the possibility of accommodating a variable number of bundles depending on their length, as evidenced in Figure 1), forming long lanes, and it can contain up to tens of thousands bundles at the same time. The different lanes are served by means of lifters (that move the bundles from the incoming shuttles up to the cranes) and upper cranes that have three different purposes:

1. To insert the incoming bundles.

2. The actual model presents many repeated subprocesses (with limited or no modifications) and similar equipment and facilities.

3. To pick-up the required outgoing bundles.

Outgoing bundles are moved again down to the ground shuttles (the same that operate the incoming flow) and are transferred to the loading stations, where the trucks are prepared for the final destinations.

This process presents some interesting complexities. Among them, it is important to highlight that:

- Bottlenecks are not known a priori and cannot be easily determined. Optimization opportunities remain almost hidden and difficult to be evaluated.

- The distributor cranes shall be programmed to avoid overlapping and to minimize the travelled distance.

- The ground shuttles, serving the warehouse both with respect to the incoming and the outgoing flows, shall be programmed carefully to optimize the two flows.

- The warehouse cranes shall be programmed to avoid overlapping, to minimize the travelled distance and to effectively allocate the bundles in order to optimize the subsequent handling stages.

As soon as the initial analysis had been completed, it emerged clearly that the process was too complex to be modelled by a standard, monolithic discrete events simulation (Figure 3). 


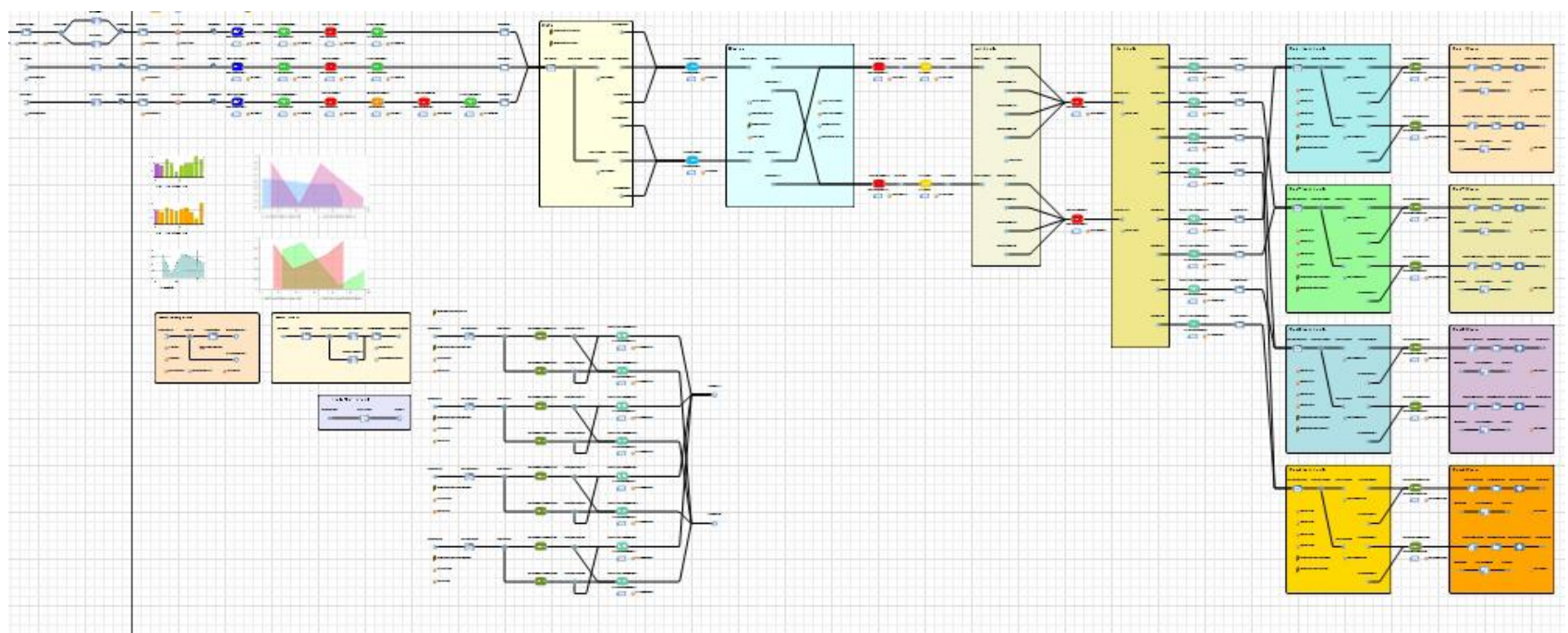

Figure 3: The main flow and its complexity (small blocks represent complex sub-processes).

Therefore, we immediately looked for a simulation environment in which we could use different methodologies and a modular approach at the same time. Thus, our final choice was that of adopting the Anylogic ${ }^{\circledR}$ Professional simulation package, that allows modellers to build applications with a fully working mix of Discrete Event Simulation, System Dynamics and Agent Based Simulation.

As stated, these requirements have been modelled making use of OOP techniques and a hierarchical modular approach aimed at simplifying the whole process. In particular, the most important elements have been carefully isolated and modelled as independent, intelligent agents that can interact and communicate with each other. These agents represent, among the various entities, the incoming and the outgoing orders, the endline machines, the lifters (those used to load-unload the underground shuttles and those used within the warehouse as well), the underground and ground shuttles, the controller stations, the middle-height distributor cranes and the warehouse cranes (Figure 4)

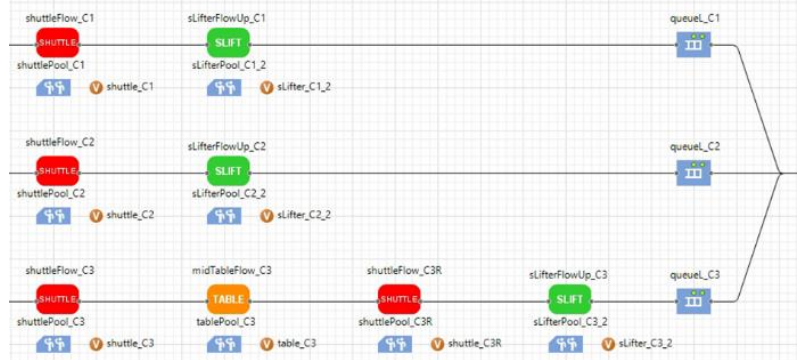

Figure 4: OOP components within the main flow (partial view)

The OOP paradigm has been extensively adopted to inherit and modify single entities, when needed. For instance, underground and ground shuttles, though differently managed, are almost similar.
Therefore, they have been derived from a common ancestor and have been later specialized to perform their own tasks adequately. The middle-height cranes and the warehouse cranes are different both with respect to the functioning and to the exterior appearance, yet they have a common parent that provide them with the basic functionalities (Figure 5 and Figure 6 show the crane class constituting elements, along with the corresponding flow management class).

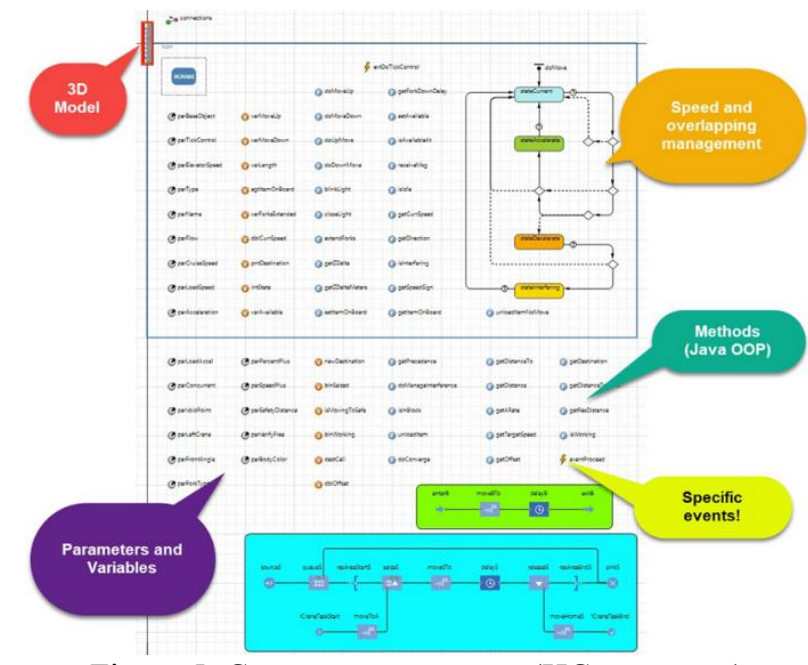

Figure 5: Crane agent structure (HCrane agent) 


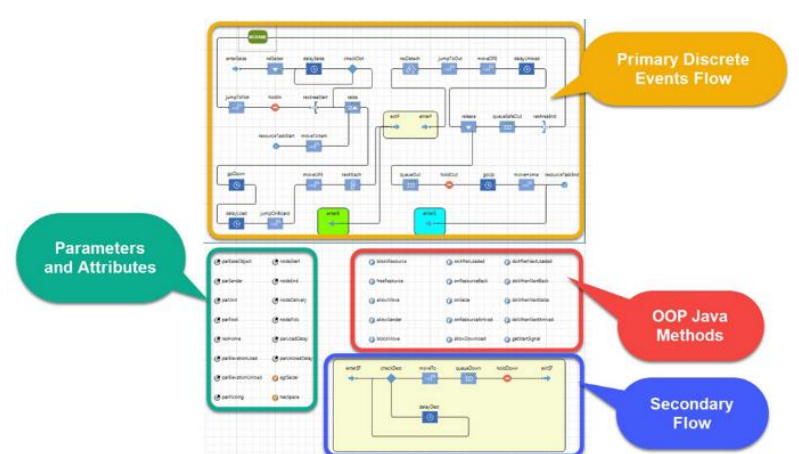

Figure 6: Crane agent flow structure (HCraneFlow agent)

\section{VERIFICATION AND VALIDATION}

One of the major concerns when modeling and simulating a real case deals with the subsequent verification and validation. Following Sargent (2011), verification ensures that the model and its implementation are correct, whereas validation grants that the model "possesses a satisfactory range of accuracy, consistent with the intended application of the model". Naturally, we may need many different experimental conditions to define the model intended applicability. Therefore, due to costs and time, it is often impossible and/or inconvenient to look for absolute validity.

Following this approach, we performed the conceptual model validation, the implementation validation and, finally, the operational validation. In particular, we used animations, events validation, and historical data to ensure that both the conceptual model and the corresponding computer translation were good enough to satisfy the end-user requirements.

Finally, we performed a set of experimental runs to optimize the parameters and a parameter-based sensitivity analysis to determine the effect of variability on the obtained outcomes. Results of this activity were good enough to ensure that the model was correct with enough degree of confidence. With respect to the available historical data, that however lacked of information about specific breakdowns and warnings from sensors (these were not available and should be collected punctually in the future), the model followed the real data with a small deviation (the error between the real and the simulated warehouse consistency resulted lower than $7.5 \%$ ) and both the performance and the saturation level of the simulated equipment was comparable with that of the real shuttles and cranes.

\section{RESULTS AND CONCLUSIONS}

In conclusion, a very complex model dealing with the automatic warehouse management of a plant for copper tubes production, has been effectively decomposed into a hierarchical, modular structure that is easy to develop, test, validate, modify and maintain. The single components that constitute the model can be effortlessly adapted to different situations and, thanks to a fully parametric approach, they can be effectively used to simplify the modelling phase and to optimize the model itself, for instance, using experiments that may vary several parameters at the same time. Further, the OOP paradigm along with the agent modelling methodology has allowed to build complex entities that are able to deal autonomously with specific, often intricate issues (for example, the cranes are able to deal with speed and overlapping control by themselves). Finally, the case study has shown that the possibility of integrating agents modelling and discrete events gives an enormous advantage in that it allows to make the most of the indepth knowledge of flows and procedure (typical of the discrete event simulation), while, at the same time, all those aspects that cannot ne mimicked with the classical discrete events programming are introduced within the agents by means of state charts and pure java OOP. The model is therefore a valuable tool to verify the current productivity of the warehouse (identifying issues and bottlenecks, for example) and to analyse potential improvements and future modifications.

\section{ACKNOWLEDGMENTS}

The authors would like to thank the Consorzio Quinn (https://www.consorzioquinn.it/) for allowing us the use of the licensed version of the Anylogic ${ }^{\circledR}$ Professional simulation package, without which this research paper would not have been possible.

\section{REFERENCES}

Armstrong D.J., 2006. The Quarks of Object-Oriented Development. Communications of the ACM, 49 (2), 123-128.

Calzavara M., Glock C.H., Grosse E.H., Persona A., Sgarbossa F. 2017. Analysis of economic and ergonomic performance measures of different rack layouts in an order picking warehouse. Computers \& Industrial Engineering, 111, 527-536.

Gu J., Goetschalckx M., McGinnis L.F., 2010. Research on warehouse design and performance evaluation: A comprehensive review. European Journal of Operational Research, 203 (3), 539-549.

Guo X., Yu Y., De Koster R.B.M. 2015. Impact of required storage space on storage policy performance in a unit-load warehouse. International Journal of Production Research, 54 (8), 2405-2418.

Kindler E., Krivy I., 2011. Object-Oriented Simulation of systems with sophisticated control. International Journal of General Systems, 40 (3), 313-343.

Krenczyk D., Davidrajuh R., Skolud B., 2019. Comparing Two Methodologies for Modeling and Simulation of Discrete-Event Based Automated Warehouses Systems. In: Hamrol A., Kujawińska A., Barraza M., eds. MANUFACTURING 2019: Advances in Manufacturing II. Cham: Springler, 161-175.

Piccinini A., Previdi F., Cimini C., Pinto R., Pirola F., 2018. Discrete event simulation for the reconfiguration of a flexible manufacturing plant. 16th IFAC Symposium on Information Control Problems in Manufacturing, pp. 465-470 June 1113, Bergamo (Italy). 
Rouwenhorst B., Reuter B., Stockrahm V., van Houtum G.J., Mantel R.J., Zijm W.H.M., 2000. Warehouse design and control: framework and literature review. European Journal of Operational Research, 122, 515-533.

Sargent R.G., 2013. Verification and validation of simulation models. Journal of Simulation, 7(1), 1224.

Smith D., Srinivas S., 2019. A Simulation-based Evaluation of Warehouse Check-in Strategies for Improving Inbound Logistics Operations. Simulation Modelling Practice and Theory, 94, 303-320.

Sumari S., Ibrahim R., Zakaria N.H., Hamid A.H.A., 2013. Comparing three simulation model using taxonomy: system dynamic simulation, discrete event simulation and agent-based simulation. International Journal of Management Excellence, 1, pp. 54-59.

Vieira A.A.C., Dias L.M.S., Pereira G.A.B., Oliveira, J.A., Carvalho M.D.S., Martins P, 2018. Simulation model generation for warehouse management: case study to test different storage strategies. International Journal of Simulation and Process Modelling, 13 (4), 324-336.

Zeigler B.P., 1987. Hierarchical, modular discrete-event modelling in an object-oriented environment. Simulation, 49 (5), 219-230.

Zeigler B.P., 1990. Object Oriented Simulation with Hierarchical Modular Models. 1st ed. Cambridge: Academic Press.

\section{AUTHORS BIOGRAPHY}

Marcello Braglia graduated (with distinction) in 1988 in Electronic Engineering at Politecnico di Milano. Since 1995, he has been a Researcher in Mechanical Technology and Production Systems at the Università degli Studi di Brescia. Since 1998, he has been employed as a researcher and since 2002, as a Full Professor, in Industrial Plants at the Università di Pisa. His research activities mainly concern maintenance, reliability, production planning, lean production, logistics and statistical quality control. He is the author of about 180 technical papers published in national and international journals and conference proceedings. He is a member of ANIMP (National Association on Industrial Plants) and AIDI (National Association of Academicians on Industrial Plants).

Marco Frosolini graduated in Mechanical Engineering at the Università di Pisa and obtained a $\mathrm{PhD}$ in Mechanical Engineering in 2005. He is currently a researcher in Industrial Plants within the same University. His research activities mainly concern equipment maintenance and reliability, production planning, logistics and project management. He is also interested in industrial information systems. He is author of more than 30 technical papers, published in national and international journals and conference proceedings.
$\mathrm{He}$ is a member of AIDI (National Association of Academicians on Industrial Plants).

Roberto Gabbrielli graduated with honours in Mechanical Engineering, specializing in Energy, at the University of Pisa (Italy). He has a PhD in Energy Power Systems and is Associate Professor in Industrial Systems Engineering at the Department of Civil and Industrial Engineering of the University of Pisa (Italy). His current research concerns production planning and control, development of decision support systems for industrial investments, occupational safety, energy saving, energy storage and the reduction of the environmental impact in industrial systems. He authored more than 25 papers published in international scientific journals.

Leonardo Marrazzini graduated (110/110) in 2016 in Mechanical Engineering at the University of Pisa. In the same year, he began his $\mathrm{PhD}$ studies in Industrial Plants within the same university. His research activities mainly concern the adaptation of Lean Manufacturing principles to engineering-to-order (ETO) production environments. His research focuses on models and techniques to support various company operations.

\section{Luca Padellini}

Luca Padellini graduated in 2018 in Mechanical Engineering at the University of Pisa. In the same year, he began his PhD studies in Smart Industry within the same University. His research activities mainly concern the digitalization of the supply chain in the Tuscan Fashion district. His research focuses on models and techniques able to improve the communication and the transfer of information between the members of the supply chain. 